\title{
BMJ Open Evaluation of the increased risk of spine fracture in patients with mood disorder compared with matched controls: a longitudinal follow-up study using a national sample cohort in Korea
}

\author{
So Young Kim, ${ }^{1}$ Chanyang Min, ${ }^{2}$ Bumjung Park, ${ }^{3}$ Miyoung Kim (D) , ${ }^{4}$ \\ Hyo Geun Choi (D) 2,3
}

To cite: Kim SY, Min C, Park B, et al. Evaluation of the increased risk of spine fracture in patients with mood disorder compared with matched controls: a longitudinal follow-up study using a national sample cohort in Korea. BMJ Open 2019;9:e027581. doi:10.1136/ bmjopen-2018-027581

- Prepublication history for this paper is available online. To view these files, please visit the journal online (http://dx.doi. org/10.1136/bmjopen-2018027581).

Received 30 0ctober 2018 Revised 31 October 2019 Accepted 11 November 2019

Check for updates

(c) Author(s) (or their employer(s)) 2019. Re-use permitted under CC BY-NC. No commercial re-use. See rights and permissions. Published by BMJ.

For numbered affiliations see end of article.

Correspondence to

Dr Hyo Geun Choi;

pupen@naver.com

\section{ABSTRACT}

Objective To evaluate the risk of spine fracture in patients with mood disorder using a nationwide cohort.

Design A longitudinal follow-up study.

Setting Claims data for the population $\geq 20$ years of age were collected from 2002 to 2013 for the Korean National Health Insurance Service-National Sample Cohort.

Participants A total of 60140 individuals with mood disorder were matched with 240560 individuals (control group) for age, sex, income, region of residence and osteoporosis.

Interventions In both the mood disorder and control groups, the history of spine fracture was evaluated. The International Classification of Diseases 10th Revision codes for mood disorder (F31-F39) and spine fracture (S220 and S320) were included.

Primary and secondary outcome measures The univariable and multivariable $\mathrm{HRs}$ and $95 \% \mathrm{Cls}$ of spine fracture for patients with mood disorder were analysed using a stratified Cox proportional hazards model. Subgroup analyses were conducted according to the history of osteoporosis, age and sex.

Results Approximately $3.3 \%(2011 / 60140)$ of patients in the mood disorder group and 2.8\% (6795/240 560) of individuals in the control group had spine fracture $(p<0.001)$. The mood disorder group demonstrated a higher adjusted HR for spine fracture than the control group (multivariable $\mathrm{HR}=1.10,95 \% \mathrm{Cl} 1.04$ to 1.15 , $p<0.001)$. The participants without osteoporosis showed a higher HR of mood disorder for spine fracture than the control participants (multivariable $\mathrm{HR}=1.25,95 \% \mathrm{Cl} 1.14$ to $1.37, p<0.001)$. According to age and sex, this result was consistent in subgroups of women aged 20-39 and 40-59 years and men aged $\geq 60$ years.

Conclusion The risk of spine fracture was increased in patients with mood disorder. The potential risk of spine fracture needs to be evaluated when managing patients with mood disorder.

\section{INTRODUCTION}

Spine fracture is the most common sign of osteoporosis and predicts the risk of subsequent fractures. ${ }^{1}$ The incidence of spine

\section{Strengths and limitations of this study}

- This study conducted a longitudinal follow-up evaluation of the risk of spine fracture in patients with mood disorder.

- The present study contributed to previous findings by using control group participants who were matched for osteoporosis and demographic factors, such as age, sex, income and region of residence, and adjusting for numerous comorbidities.

- In addition, subgroup analyses were conducted to examine the risk of spine fracture in patients with mood disorder according to the presence of osteoporosis, age and sex.

- Although International Classification of Diseases 10th Revision codes are based on a diagnosis made by a physician, they lack information on the severity of disease and treatment history.

- Although the number of variables was considered, there were potential confounding effects due to unconsidered variables.

fracture is heterogeneous according to how it is defined and patient ethnicity. ${ }^{2}$ In the USA, approximately 707 per 100000 men and 1083 per 100000 women suffer from spine fracture. Korean individuals have a high incidence of spine fracture, which affects approximately 544 per 100000 men and 1575 per 100000 women. ${ }^{3}$ The incidence of spine fracture is increasing due to ageing of the population. ${ }^{2}$ However, spine fracture is often underdiagnosed and undertreated. It has been estimated that approximately two-thirds to three-quarters of spine fractures are asymptomatic. ${ }^{4}$ Only one-quarter to one-third of spine fractures are clinically recognised. Because spine fracture significantly worsens patient mortality and quality of life, the risk assessment and early detection of spine fractures are crucial. ${ }^{5}$ In addition to 
osteoporosis and ageing, several comorbidities, including diabetes ${ }^{6}{ }^{6}$ hypertension, ${ }^{7}$ dyslipidaemia ${ }^{8}$ and ischaemic heart disease, ${ }^{9}$ have been proposed to be associated with fractures. Moreover, physical disabilities and susceptibility to falls increase the susceptibility to spine fractures. ${ }^{10}$

Depression is a prevalent disorder that affects approximately $2 \%-15 \%$ of the general population. ${ }^{11}$ Multiple factors, including both genetic and environmental factors, are related to depression. ${ }^{12}$ Thus, several medical disorders, such as osteoporosis and neurodegenerative disorders, have been suggested to be associated with depressive disorders. ${ }^{13} 14$ In accordance with this finding, several previous studies have reported an increased risk of osteoporotic fracture in depressed patients. ${ }^{15}{ }^{16} \mathrm{High}$ risks of osteoporosis and falls may mediate the increased risk of fracture in depressed patients. ${ }^{16}{ }^{17}$ Moreover, accidental traumatic fractures may influence the relationship between depression and fractures. However, spine fracture is associated with a lesser degree of trauma than other osteoporotic fractures; thus, acute traumatic fractures may contribute less to the incidence of spine fracture than other fracture types. Thus, the association between depression and spine fracture may be different from its association with other types of fractures. A prior study demonstrated the association of osteoporotic thoracolumbar fracture with depression in postmenopausal women. ${ }^{17}$ Only a few previous studies have proposed a high risk of spine fracture in patients with depressive disorder. ${ }^{18}$ However, comorbid conditions were not sufficiently matched between the study and control groups. Because both mood disorders, including depression, and spine fracture are associated with several medical disorders, these possible confounders must be addressed to estimate the risk of spine fracture in patients with mood disorder.

We hypothesised that mood disorder may increase the risk of new-onset spine fractures independent of other comorbid conditions. To investigate the risk of spine fracture in patients with mood disorder according to age and sex, subgroup analyses were performed in this study. Few prior studies have evaluated the differential risk of spine fracture in patients with mood disorder according to the population characteristics of age and sex.

\section{MATERIALS AND METHODS}

\section{Patient and public involvement}

This study did not involve patients. This national cohort study relied on data from the Korean Health Insurance Review and Assessment Service-National Sample Cohort. These data were described in detail in our previous studies. ${ }^{19} 20$ The Korean National Health Insurance Service (NHIS) selects samples directly from the entire population database to prevent non-sampling errors. Approximately $2 \%$ of the samples ( 1 million) were selected from the entire Korean population ( 50 million). This study was not based on the survey or interventions.
Instead, the registered health claim codes were used to investigate medical histories.

\section{Participant selection}

Of a total of 1125691 patients with 114369638 medical claim codes, we included participants who were diagnosed with mood disorder. Mood disorder was defined using International Classification of Diseases 10th Revision (ICD-10) codes F31 (bipolar affective disorder) through F39 (unspecified mood disorder) as diagnosed by a psychiatrist between 2002 and 2013. Among patients with these ICD-10 codes, we selected the participants who were treated $\geq 2$ times $(n=68019)$.

Spine fracture was defined as a fracture of a thoracic vertebra (ICD-10 code: S220) or a lumbar vertebra (S320) between 2002 and 2013 ( $\mathrm{n}=23$ 026). As in previous studies, fractures of cervical vertebrae were excluded due to the rare incidence of these fractures. ${ }^{21}$

Mood disorder participants were matched 1:4 with participants (control group) who were never diagnosed with mood disorder between 2002 and 2013 among this cohort. The control group was selected from the mother population ( $n=1091$ 119). The participants were matched for age group, sex, income group, region of residence and history of osteoporosis. To prevent selection bias when choosing the matched participants, the control group participants were sorted using random numbers, and selection began sequentially from the top of the list. We set the index data as the date of the diagnosis of mood disorder. It was assumed that each matched control participant was involved at the same time as each matched mood disorder participant (index date). Therefore, participants in the control group who died before the index date were excluded. We excluded participants who were diagnosed with malignant neoplasms of the bone and articular cartilage $(n=32)$. In both the mood disorder and control groups, participants with a history of spine fracture before the index date were excluded. In the mood disorder group, 1356 participants were excluded. Patients with mood disorder for whom we could not identify enough matching participants were excluded $(\mathrm{n}=12)$. We excluded patients younger than 20 years old $(\mathrm{n}=6479)$. Finally, 1:4 matching resulted in the inclusion of $60140 \mathrm{mood}$ disorder participants and $240560 \mathrm{control}$ participants (figure 1).

\section{Variables}

The age groups were classified using 5 -year intervals: $20-24,25-29,30-34 \ldots$ and $85+$ yearsold. A total of 14 age groups were designated. The income groups were initially divided into 41 classes ( 1 health aid class, 20 selfemployment health insurance classes and 20 employment health insurance classes). These groups were recategorised into five classes (class 1 (lowest income) to class 5 (highest income)). The health insurance population was grouped according to 10 deciles. This recategorisation was performed by the National Institute of Health Science to deidentify the participants. Participants' 


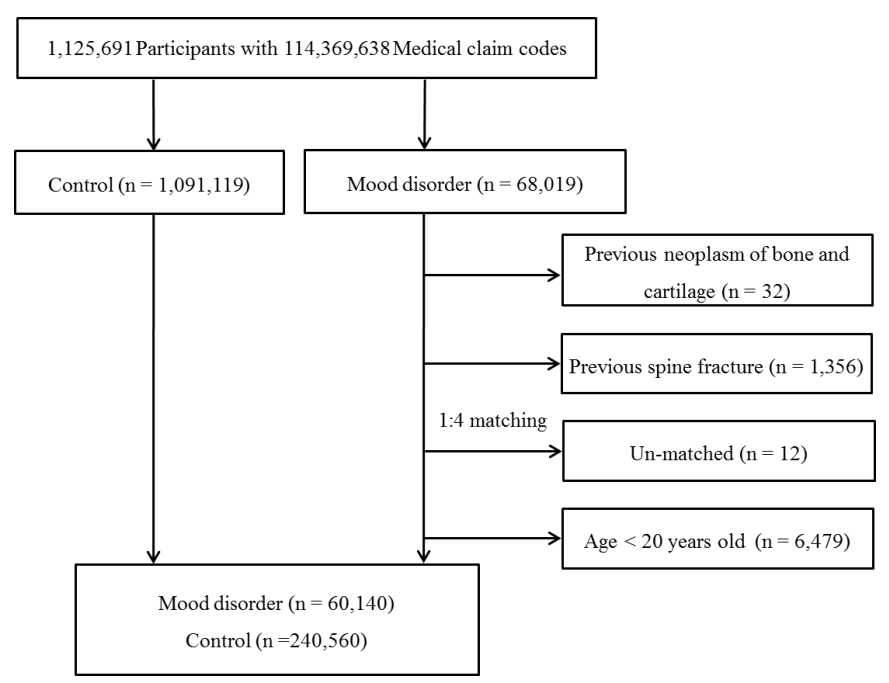

Figure 1 Schematic illustration of the participant selection process that was used in the present study. Of a total of 1125691 participants, 60140 patients with mood disorder were matched with 240560 control participants by age, group, sex, income group, region of residence and osteoporosis.

regions of residence were divided into 16 areas according to administrative districts. These regions were regrouped into urban (Seoul, Busan, Daegu, Incheon, Gwangju, Daejeon and Ulsan) and rural (Gyeonggi, Gangwon, Chungcheongbuk, Chungcheongnam, Jeollabuk, Jeollanam, Gyeongsangbuk, Gyeongsangnam and Jeju) areas.

Participants diagnosed with osteoporosis between 2002 and 2013 were diagnosed according to the following ICD-10 codes: M80 (osteoporosis with pathological fracture), M81 (osteoporosis without pathological fracture) and M82 (osteoporosis associated with diseases classified elsewhere). Among the identified patients with osteoporosis, we selected those who were treated $\geq 2$ times or who were diagnosed with osteoporosis by bone density testing using X-rays or CT (claim code: E7001-E7004, HC341-HC345) between 2002 and 2013. Alzheimer's dementia was defined if the participants were diagnosed with Alzheimer's disease (G30) or dementia in Alzheimer's disease (F00). For the accuracy of diagnosis, we selected only participants treated $\geq 2$ times. The Charlson Comorbidity Index (CCI) was used for 17 comorbidities as the continuous variable (0 (no comorbidity) through 29 (multiple comorbidities)). ${ }^{22}$

\section{Statistical analyses}

The $\chi^{2}$ test was used to compare the rate of general characteristics between participants in the mood disorder and control groups.

To analyse the HR of mood disorder on spine fracture, a stratified Cox proportional hazards model was used. In this analysis, univariable (simple) and multivariable (Alzheimer's dementia and CCI scores) models were used. In these analyses, age, sex, income, region of residence and history of osteoporosis were stratified. KaplanMeier analysis and the log-rank test were used.
For the subgroup analysis, we divided the participants by age (20-39, 40-59 and 60+ years old) and sex (male and female) to confirm that these relations were reliable according to age and sex, because both spine fracture and mood disorder are influenced by age and sex. The age and sex subgroups were divided into tertiles. Additionally, we divided the participants according to their history of osteoporosis to confirm that these associations were consistent with/without osteoporosis.

Two-tailed analyses were conducted, and $\mathrm{p}$ values less than 0.05 were considered to indicate significance. The results were statistically analysed using SPSS V.21.0 (IBM).

\section{RESULTS}

Approximately 3.3\% (2011/60 140) and 2.5\% (6795/240 560) of participants in the mood disorder and control groups, respectively, had spine fractures $(\mathrm{p}<0.001)$ (table 1). Age, sex, income, region of residence and osteoporosis were matched between the mood disorder and control groups. The CCI score and Alzheimer's dementia were different between the mood disorder and control groups (each $\mathrm{p}<0.001)$.

The multivariable HR for spine fracture was 1.10 times higher in the mood disorder group than in the control group $(95 \%$ CI 1.04 to $1.15, \mathrm{p}<0.001)$ (table 2 and figure 2).

In the subgroup analysis according to the presence of osteoporosis, participants without osteoporosis demonstrated a higher HR of mood disorder for spine fracture than participants with osteoporosis (multivariable $\mathrm{HR}=1.25,95 \%$ CI 1.14 to $1.37, \mathrm{p}<0.001)$.

According to age and sex, the subgroup of young women, but not the subgroup of young men, demonstrated a higher adjusted HR than the control group (adjusted $\mathrm{HR}=1.47$, 95\% CI 1.06 to 2.03, $\mathrm{p}=0.021$ ) (table 3). The subgroup of middle-aged women, but not the subgroup of middle-aged men, had a higher adjusted $\mathrm{HR}$ than the control group (multivariable $\mathrm{HR}=1.14$, $95 \%$ CI 1.00 to $1.30, \mathrm{p}=0.049)$. The elderly men, but not the elderly women, demonstrated a higher adjusted HR than the control group (multivariable $\mathrm{HR}=1.27$, 95\% CI 1.10 to $1.47, \mathrm{p}<0.001)$.

\section{DISCUSSION}

The risk of spine fracture was 1.10 times higher in the mood disorder group than in the control group in the present study. In the mood disorder group, the participants without osteoporosis demonstrated a 1.25 times higher risk of spine fracture than the participants with osteoporosis. According to age and sex, women 20-39 and 40-59 years old and men $\geq 60$ years old in the mood disorder group demonstrated a high risk of spine fracture. Only a few previous studies have reported an increased risk of spine fracture in patients with mood disorder. The present study contributed to previous findings by using control group participants who were matched for osteoporosis and demographic factors, 
Table 1 General characteristics of the participants Total participants

\begin{tabular}{|c|c|c|c|}
\hline \multirow[b]{2}{*}{ Characteristic } & \multicolumn{3}{|c|}{ Total participants } \\
\hline & $\begin{array}{l}\text { Mood disorder, } \\
\text { n (\%) }\end{array}$ & Control, n (\%) & $P$ value \\
\hline Age (years) & & & 1.000 \\
\hline $20-24$ & $3880(6.5)$ & $15520(6.5)$ & \\
\hline $25-29$ & 4595 (7.6) & $18380(7.6)$ & \\
\hline $30-34$ & 5269 (8.8) & 21076 (8.8) & \\
\hline 35-39 & 5799 (9.6) & $23196(9.6)$ & \\
\hline $40-44$ & $6160(10.2)$ & $24640(10.2)$ & \\
\hline $45-49$ & $6443(10.7)$ & $25772(10.7)$ & \\
\hline $50-54$ & 6147 (10.2) & 24588 (10.2) & \\
\hline $55-59$ & 4995 (8.3) & 19980 (8.3) & \\
\hline $60-64$ & $4628(7.7)$ & $18512(7.7)$ & \\
\hline $65-69$ & 4419 (7.3) & $17676(7.3)$ & \\
\hline $70-74$ & $3677(6.1)$ & $14708(6.1)$ & \\
\hline $75-79$ & $2298(3.8)$ & 9192 (3.8) & \\
\hline $80-84$ & $1217(2.0)$ & $4868(2.0)$ & \\
\hline $85+$ & $613(1.0)$ & $2452(1.0)$ & 1.000 \\
\hline \multicolumn{4}{|l|}{ Sex } \\
\hline Male & 20601 (34.3) & 82404 (34.3) & \\
\hline Female & 39539 (65.7) & $158156(65.7)$ & \\
\hline Income & & & 1.000 \\
\hline 1 (lowest) & $9421(15.7)$ & $37684(15.7)$ & \\
\hline 2 & $8922(14.8)$ & $35688(14.8)$ & \\
\hline 3 & 10379 (17.3) & 41516 (17.3) & \\
\hline 4 & 12933 (21.5) & $51732(21.5)$ & \\
\hline 5 (highest) & 18485 (30.7) & $73940(30.7)$ & \\
\hline \multicolumn{2}{|c|}{ Region of residence } & & 1.000 \\
\hline Urban & 27608 (45.9) & $110432(45.9)$ & \\
\hline Rural & 32532 (54.1) & 130128 (54.1) & \\
\hline Osteoporosis & 13412 (22.3) & 53648 (22.3) & 1.000 \\
\hline CCl score* & & & $<0.001 \dagger$ \\
\hline 0 & 17995 (29.9) & $108159(45.0)$ & \\
\hline 1 & $5767(9.6)$ & 26552 (11.0) & \\
\hline$\geq 2$ & $36378(60.5)$ & $105849(44.0)$ & \\
\hline $\begin{array}{l}\text { Alzheimer's } \\
\text { dementia }\end{array}$ & $5364(8.9)$ & 8665 (3.6) & $<0.001 \dagger$ \\
\hline Spine fracture & 2011 (3.3) & 6795 (2.8) & $<0.001 \dagger$ \\
\hline
\end{tabular}

*The Charlson Comorbidity Index was calculated.

$+\chi^{2}$ test; significance at $p<0.05$.

such as age, sex, income and region of residence. In addition, the subgroup analysis demonstrated an increased risk of spine fracture in patients with mood disorder without osteoporosis.

Similar to our results, the results of previous studies have demonstrated a high risk of spine fracture in depressed patients. The incidence of vertebral fracture was 1.41 times higher in depressed patients than in control group participants in a population cohort study (95\% CI 1.26 to $1.57, \mathrm{p}<0.001) .{ }^{18}$ However, in the same study, the incidence of a medical history of another disease was higher in depressed patients than in the controls. Although the authors adjusted for participants' medical histories, these medical histories could confound the impact of depression on vertebral fracture. Another longitudinal follow-up study reported a higher incidence of thoracolumbar fractures in depressed participants than in control group participants $(35.4 \%$ vs $25.1 \%, \mathrm{p}<0.05) .{ }^{17}$ However, the study population was restricted to postmenopausal women. On the other hand, this study presented the risk of spine fracture in patients with mood disorder in an adult population compared with that in control group participants who were matched and adjusted for their medical histories.

Decreased bone mineral density in patients with mood disorder may increase the risk of osteoporotic fractures, including spine fractures. ${ }^{23-25}$ Several aspects of humoral and behavioural changes in depressed patients may influence the occurrence of spine fracture. A dysregulated endocrine system, such as the hypothalamic-pituitary axis, in depressed patients may accelerate osteoporotic bone metabolism. ${ }^{26}$ Depressed patients have high cortisol levels due to abnormal activation of the hypothalamicpituitary axis. High cortisol is known to decrease bone mineral density. ${ }^{26}$ In terms of behavioural aspects, the physical inactivity of depressed patients might reduce bone mineral density. ${ }^{27} \mathrm{~A}$ case-control study reported that physical activity was correlated with osteoresorption markers in premenopausal women with unipolar depression. ${ }^{27}$ Moreover, antidepressant medication may also lead to decreased bone mineral density. ${ }^{2829}$ A populationbased, cross-sectional study demonstrated that bone mineral density was $6.1 \%$ lower in selective serotonin reuptake inhibitor users than in non-users $(p=0.016) .{ }^{28}$ An animal study demonstrated that osteoblast differentiation and mineralisation were inhibited by fluoxetine, thereby resulting in delayed bone regeneration. ${ }^{30}$

In the subgroup analysis according to the presence of osteoporosis, the participants in the mood disorder group without osteoporosis demonstrated a higher risk of spine fracture than those with osteoporosis in this study. This implies that there might be other contributing factors in addition to osteoporosis for the association between mood disorder and the risk of spine fracture. Impaired balance function in depressed patients may also increase the risk of spine fracture. Depressive symptoms have been shown to worsen balance function, as measured using postural sway tests in elderly patients. ${ }^{31}$ Impaired balance and executive functions in depressed patients have been demonstrated in several studies. ${ }^{31}{ }^{32}$ Reduced executive function was demonstrated in depressed patients in a functional MRI study, which showed altered frontolimbic brain cortico-subcortical connectivity in these patients. ${ }^{32}$ In addition, the brain areas related to balance function, such as the basal ganglia and limbic circuits, are impaired in depressed patients. ${ }^{31}$ In addition to impaired balance 
Table 2 Crude and adjusted HR $(95 \% \mathrm{Cl})$ of mood disorder for spine fracture

\begin{tabular}{|c|c|c|c|c|}
\hline \multirow[b]{2}{*}{ Characteristic } & \multicolumn{4}{|l|}{ HR $(95 \% \mathrm{Cl})$} \\
\hline & Crude* $^{*}$ & $P$ value & 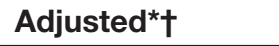 & $P$ value \\
\hline \multicolumn{5}{|l|}{ Total $(n=300700)$} \\
\hline Control & 1.00 & & 1.00 & \\
\hline \multicolumn{5}{|c|}{ Participants with osteoporosis $(n=67060)$} \\
\hline Control & 1.00 & & 1.00 & \\
\hline \multicolumn{5}{|c|}{ Participants without osteoporosis $(n=233640)$} \\
\hline Mood disorder & $1.45(1.32$ to 1.59$)$ & $<0.001 \ddagger$ & $1.25(1.14$ to 1.37$)$ & $<0.001 \ddagger$ \\
\hline Control & 1.00 & & 1.00 & \\
\hline
\end{tabular}

*Stratified the model for age, income, region of residence and osteoporosis.

†Adjusted the model for the Charlson Comorbidity Index and history of Alzheimer's dementia.

$\ddagger$ Cox proportional hazards regression model; significance at $p<0.05$.

function, depression may increase the risk of falls, especially in the elderly population. ${ }^{33}$ Last, an unhealthy lifestyle in depressed patients may increase the risk of spine fracture or aggravate comorbid physical conditions, such as metabolic diseases, stroke and cardiovascular disorders. ${ }^{35}$

In the subgroup analyses according to sex, female patients with mood disorder aged $20-39$ and $40-59$ years demonstrated a high risk of spine fracture in this study. This can be explained by the high incidence of both mood disorder and osteoporosis in women compared with men. ${ }^{33}$ In addition, the high risk of falls in female patients with mood disorder may mediate the increased risk of spine fracture. A previous study reported that the risk of falls in depressed patients was higher in women than in men. ${ }^{33}$ Moreover, spine fractures are caused by relatively low-trauma injuries compared with other osteoporotic fractures. Thus, women may be more affected by spine fractures than men. ${ }^{36}$ In men, high-trauma fractures, such as hip fractures, are 3.12 times more frequent than those in women (95\% CI 1.70 to 5.71). ${ }^{37}$ Among participants in the mood disorder group, in the old age group, the men subgroup showed an increased risk of spine fracture in this study. In the old age group, the rates of mood disorder and osteoporosis were increased in both the men and women subgroups. Thus, the effects of

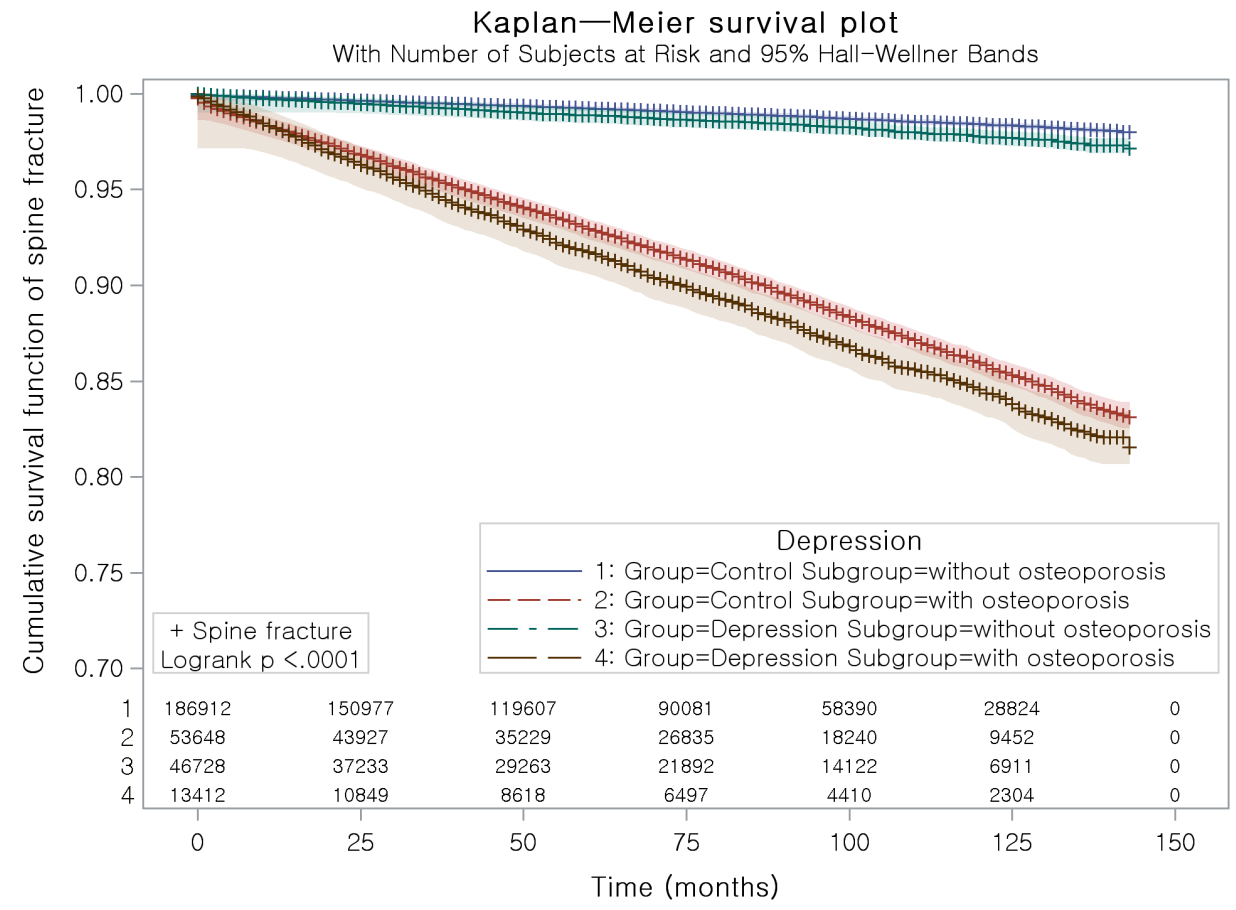

Figure 2 Kaplan-Meier curve of spine fracture for depression. This was explained as a survival function curve. 
Table 3 Subgroup analysis of the crude and adjusted HR $(95 \% \mathrm{Cl})$ of mood disorder for spine fracture according to age and sex

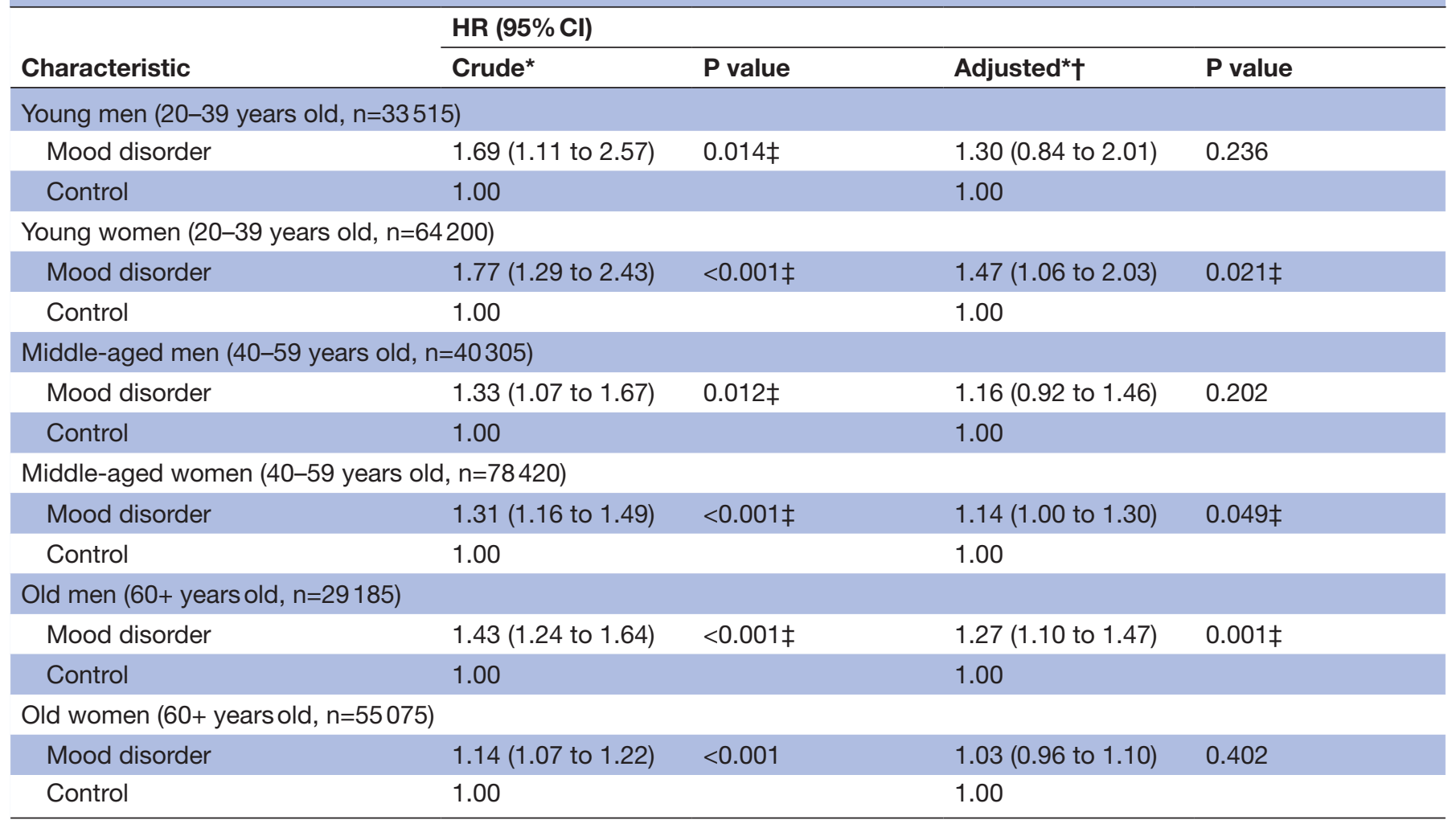

*Stratified the model for age, income, region of residence and osteoporosis.

†Adjusted the model for the Charlson Comorbidity Index and history of Alzheimer's dementia.

$\ddagger$ Cox proportional hazards regression model; significance at $p<0.05$.

mood disorder and osteoporosis on spine fracture might be increased in older men.

There are several merits of this study. The present study was based on a nationwide representative population that was verified in a prior study. ${ }^{38}$ The NHIS data include all Korean citizens without exception. The control group was matched for age, sex, income, region of residence and osteoporosis. Because socioeconomic factors can influence the availability of medical care, income and region of residence were matched between participants in the mood disorder and control groups. Moreover, random sampling was performed to minimise selection bias in the control group. Although Alzheimer's dementia is one of the related factors for both spine fracture and mood disorder, it was not matched in this study because strict matching increased the rate of participant dropout due to a lack of control participants. To attenuate the confounding effects of Alzheimer's dementia and other medical histories, Alzheimer's dementia and the CCI were adjusted for in this study.

On the other hand, a few weaknesses should be considered when interpreting the current results. The ICD-10 codes for mood disorder and spine fracture were used for this study. Although ICD-10 codes are based on a diagnosis made by a physician, they lack information on the severity of disease and treatment history. Because approximately
$12.9 \%$ of patients with radiographically detected spine fractures underwent medical treatment, an underdiagnosis of vertebral fracture may have led to an underestimation of the risk of vertebral fracture in patients with mood disorder in the present study. ${ }^{39}$ Similarly, patients with mood disorder or osteoporosis who were not diagnosed or treated might have been missed in this study. To minimise selection bias due to medical accessibility, the present study matched the mood disorder and control groups for the socioeconomic factors income and region of residence. In addition, medications used to treat mood disorder and spine fractures may have influenced the impact of mood disorder on the incidence of spine fractures. Antidepressant medication is known to increase the risk of fracture $(\mathrm{OR}=1.66,95 \% \mathrm{CI} 1.42$ to 1.95$) .{ }^{40}$ Although demographic and socioeconomic factors were matched between the mood disorder and control groups, those in the mood disorder group were more clinically severe than those in the control group, with a higher rate of Alzheimer's disease and a higher CCI. To minimise the confounding effects of these morbidities, Alzheimer's disease and the CCI were adjusted. Finally, other comorbid conditions, including alcohol use, smoking and obesity, were not evaluated in this study. These lifestyle factors may impact the risk of fracture via an increased risk of cardiovascular disease. In addition, smoking was 
reported to increase the risk of spine fracture 2.50 times (95\% CI 1.58 to 3.95 ), which was probably due to the decreased trabecular bone turnover rate ${ }^{41}$ Future studies are warranted to evaluate the influence of these factors.

\section{CONCLUSION}

The risk of spine fracture was increased in adult patients with mood disorder. In the mood disorder group, participants without osteoporosis showed an increased risk of spine fracture. According to age and sex, women aged 20-39 and 40-59years and men aged $\geq 60$ years with mood disorder demonstrated a higher risk of spine fracture than participants in the control group.

\section{Author affiliations}

${ }^{1}$ Department of Otorhinolaryngology-Head \& Neck Surgery, CHA Bundang Medical Center, CHA University, Seongnam, South Korea

${ }^{2}$ Hallym Data Science Laboratory, Hallym University College of Medicine, Chuncheon, South Korea

${ }^{3}$ Otorhinolaryngology-Head and Neck Surgery, Hallym University College of Medicine, Chuncheon, South Korea

${ }^{4}$ Laboratory Medicine, Hallym University College of Medicine, Chuncheon, South Korea

Contributors HGC designed the study. HGC, CM, BP and MK analysed the data. SYK and HGC drafted and revised the paper. All authors approved the final version of the manuscript.

Funding This research was supported by funding from the National Research Foundation (NRF) of Korea (NRF-2018-R1D1A1A02085328 and NRF-2017R1C1B1007696).

Competing interests None declared.

Patient consent for publication Not required.

Ethics approval The Ethics Committee of Hallym University (2017-1102) approved the use of these data. The requirement for written informed consent was waived by the institutional review board.

Provenance and peer review Not commissioned; externally peer reviewed.

Data availability statement Releasing of the data by the researcher is not allowed legally. All of data are available from the database of National health Insurance Sharing Service (NHISS) https://nhiss.nhis.or.kr/ NHISS allows all of this data for the any researcher who promises to follow the research ethics with some cost. If you want to access the data of this article, you could download it from the website after promising to follow the research ethics.

Open access This is an open access article distributed in accordance with the Creative Commons Attribution Non Commercial (CC BY-NC 4.0) license, which permits others to distribute, remix, adapt, build upon this work non-commercially, and license their derivative works on different terms, provided the original work is properly cited, appropriate credit is given, any changes made indicated, and the use is non-commercial. See: http://creativecommons.org/licenses/by-nc/4.0/.

\section{ORCID iDs}

Miyoung Kim http://orcid.org/0000-0002-8903-5044

Hyo Geun Choi http://orcid.org/0000-0003-1655-9549

\section{REFERENCES}

1. Ensrud KE, Schousboe JT. Clinical practice. vertebral fractures. $N$ Engl J Med 2011;364:1634-42.

2. Ballane G, Cauley JA, Luckey MM, et al. Worldwide prevalence and incidence of osteoporotic vertebral fractures. Osteoporos Int 2017;28:1531-42.

3. Shin CS, Kim MJ, Shim SM, et al. The prevalence and risk factors of vertebral fractures in Korea. J Bone Miner Metab 2012;30:183-92.
4. Fink HA, Milavetz DL, Palermo L, et al. What proportion of incident radiographic vertebral deformities is clinically diagnosed and vice versa? J Bone Miner Res 2005;20:1216-22.

5. Kwon H-Y, Ha Y-C, Yoo J-I. Health-Related quality of life in accordance with fracture history and comorbidities in Korean patients with osteoporosis. J Bone Metab 2016;23:199-206.

6. Lin Y-C, Wu J, Kuo S-F, et al. Vertebral fractures in type 2 diabetes patients: utility of trabecular bone score and relationship with serum bone turnover biomarkers. Journal of Clinical Densitometry 2019.

7. Yang S, Nguyen ND, Center JR, et al. Association between hypertension and fragility fracture: a longitudinal study. Osteoporos Int 2014;25:97-103.

8. Kaji H, Hisa I, Inoue Y, et al. Low density lipoprotein-cholesterol levels affect vertebral fracture risk in female patients with primary hyperparathyroidism. Exp Clin Endocrinol Diabetes 2010;118:371-6.

9. Paccou J, D'Angelo S, Rhodes A, et al. Prior fragility fracture and risk of incident ischaemic cardiovascular events: results from UK Biobank. Osteoporos Int 2018;29:1321-8.

10. Domiciano DS, Machado LG, Lopes JB, et al. Incidence and risk factors for osteoporotic vertebral fracture in low-income communitydwelling elderly: a population-based prospective cohort study in Brazil. The São Paulo Ageing \& Health (SPAH) Study. Osteoporos Int 2014;25:2805-15.

11. Moussavi S, Chatterji S, Verdes E, et al. Depression, chronic diseases, and decrements in health: results from the world health surveys. The Lancet 2007;370:851-8.

12. Lesch KP. Gene-Environment interaction and the genetics of depression. J Psychiatry Neurosci 2004;29:174-84.

13. Anisman H, Merali Z, Hayley S. Neurotransmitter, peptide and cytokine processes in relation to depressive disorder: comorbidity between depression and neurodegenerative disorders. Prog Neurobiol 2008;85:1-74.

14. Drosselmeyer J, Rapp MA, Hadji P, et al. Depression risk in female patients with osteoporosis in primary care practices in Germany. Osteoporos Int 2016;27:2739-44.

15. Williams LJ, Pasco JA, Jackson $\mathrm{H}$, et al. Depression as a risk factor for fracture in women: a 10 year longitudinal study. J Affect Disord 2016;192:34-40.

16. Wu Q, Liu J, Gallegos-Orozco JF, et al. Depression, fracture risk, and bone loss: a meta-analysis of cohort studies. Osteoporos Int 2010;21:1627-35.

17. Whooley MA, Kip KE, Cauley JA, et al. Depression, falls, and risk of fracture in older women. study of osteoporotic fractures Research Group. Arch Intern Med 1999;159:484-90.

18. Zong $Y$, Tang $Y, X u e ~ Y$, et al. Depression is associated with increased incidence of osteoporotic thoracolumbar fracture in postmenopausal women: a prospective study. Eur Spine J 2016;25:3418-23.

19. Lee S-C, Hu L-Y, Huang M-W, et al. Risk of vertebral fracture in patients diagnosed with a depressive disorder: a nationwide population-based cohort study. Clinics 2017;72:44-50.

20. Kim SY, Kim H-J, Lim H, et al. Bidirectional association between gastroesophageal reflux disease and depression: two different nested case-control studies using a national sample cohort. Sci Rep 2018;8:11748.

21. Kim SY, Lim J-S, Kong IG, et al. Hearing impairment and the risk of neurodegenerative dementia: a longitudinal follow-up study using a national sample cohort. Sci Rep 2018;8:15266.

22. Olmos JM, Hernández JL, Martínez J, et al. Prevalence of vertebral fracture and densitometric osteoporosis in Spanish adult men: the Camargo cohort study. J Bone Miner Metab 2018;36:103-10.

23. Quan H, Li B, Couris CM, et al. Updating and validating the Charlson comorbidity index and score for risk adjustment in hospital discharge Abstracts using data from 6 countries. Am J Epidemiol 2011;173:676-82.

24. Hlis RD, Mclntyre RS, Maalouf NM, et al. Association between bone mineral density and depressive symptoms in a population-based sample. J Clin Psychiatry 2018;79.

25. Mollard E, Bilek L, Waltman N. Emerging evidence on the link between depressive symptoms and bone loss in postmenopausal women. Int J Womens Health 2018;10:1-9.

26. Schweiger JU, Schweiger U, Hüppe $\mathrm{M}$, et al. Bone density and depressive disorder: a meta-analysis. Brain Behav 2016;6:e00489.

27. Altindag $\mathrm{O}$, Altindag $\mathrm{A}$, Asoglu $\mathrm{M}$, et al. Relation of cortisol levels and bone mineral density among premenopausal women with major depression. Int J Clin Pract 2007;61:416-20.

28. Petronijević M, Petronijević N, Ivković M, et al. Low bone mineral density and high bone metabolism turnover in premenopausal women with unipolar depression. Bone 2008;42:582-90.

29. Feuer AJ, Demmer RT, Thai A, et al. Use of selective serotonin reuptake inhibitors and bone mass in adolescents: an NHANES study. Bone 2015;78:28-33. 
30. Weaver SR, Hernandez LL. Could use of selective serotonin reuptake inhibitors during lactation cause persistent effects on maternal bone? J Mammary Gland Biol Neoplasia 2018;23:5-25.

31. Bradaschia-Correa V, Josephson AM, Mehta D, et al. The selective serotonin reuptake inhibitor fluoxetine directly inhibits osteoblast differentiation and mineralization during fracture healing in mice. $J$ Bone Miner Res 2017;32:821-33.

32. Pieruccini-Faria F, Muir-Hunter SW, Montero-Odasso M. Do depressive symptoms affect balance in older adults with mild cognitive impairment? Results from the "gait and brain study". Exp Gerontol 2018;108:106-11.

33. Liao C, Feng Z, Zhou D, et al. Dysfunction of fronto-limbic brain circuitry in depression. Neuroscience 2012;201:231-8.

34. Park Y, Paik N-J, Kim KW, et al. Depressive symptoms, falls, and fear of falling in old Korean adults: the Korean longitudinal study on health and aging (KLoSHA). J Frailty Aging 2017;6:144-7.

35. Casteran M, Putot A, Pfitzenmeyer F, et al. Analysis of the impact of a cognitive task on the posture of elderly subjects with depression compared with healthy elderly subjects. Clin Neurophysiol 2016;127:3406-11.
36. Park SJ, Hong S, Jang H, et al. The prevalence of chronic physical diseases comorbid with depression among different sex and age groups in South Korea: a population-based study, 2007-2014. Psychiatry Investig 2018;15:370-5.

37. SH O, Kim D, Lee YE, et al. Comparison of screening strategies for prevalent vertebral fractures in South Korea: vertebral fracture assessment vs. spine radiography. BMC Musculoskelet Disord 2018;19.

38. Ensrud KE, Blackwell TL, Cawthon PM, et al. Degree of trauma differs for major osteoporotic fracture events in older men versus older women. J Bone Miner Res 2016;31:204-7.

39. Lee J, Lee JS, Park S-H, et al. Cohort profile: the National health insurance Service-National sample cohort (NHIS-NSC), South Korea. Int J Epidemiol 2017;46:e15.

40. Gehlbach SH, Bigelow C, Heimisdottir M, et al. Recognition of vertebral fracture in a clinical setting. Osteoporos Int 2000;11:577-82.

41. Thorell K, Ranstad K, Midlöv P, et al. Is use of fall risk-increasing drugs in an elderly population associated with an increased risk of hip fracture, after adjustment for multimorbidity level: a cohort study. BMC Geriatr 2014;14:131. 